\title{
Atrial Septal Defect Tracking in 3D Cardiac Ultrasound
}

\author{
Marius George Linguraru ${ }^{1}$, Nikolay V.Vasilyev², \\ Pedro J. del Nido ${ }^{2}$, and Robert D. Howe ${ }^{1}$ \\ ${ }^{1}$ Division of Engineering and Applied Sciences, Harvard University, Cambridge, MA, USA \\ ${ }^{2}$ Dept. of Cardiac Surgery, Children's Hospital, Harvard Medical School, Boston, MA, USA \\ mglin@deas.harvard.edu
}

\begin{abstract}
We are working to develop beating-heart atrial septal defect (ASD) closure techniques using real-time 3-D ultrasound guidance. The major image processing challenges are the low image quality and the high frame rate. This paper presents comparative results for ASD tracking in sequences of 3D cardiac ultrasound. We introduce a block flow technique, which combines the velocity computation from optical flow for an entire block with template matching. Enforcing similarity constraints to both the previous and first frames ensures optimal and unique solutions. We compare the performance of the proposed algorithm with that of block matching and optical flow on six in-vivo 4D datasets acquired from porcine beating-heart procedures. Results show that our technique is more stable and has higher sensitivity than both optical flow and block matching in tracking ASDs. Computing velocity at the block level, our technique is much faster than optical flow and comparable in computation cost to block matching.
\end{abstract}

\section{Introduction}

Atrial septal defects (ASD) are congenital heart malformations consisting of openings in the septum between the atria. This allows blood to shunt from the left atrium into the right atrium, which decreases the efficiency of heart pumping. Although the surgical intervention for ASD closure is well established and has excellent prognosis, it is performed under cardiopulmonary bypass (CPB), which has widely acknowledged harmful effects. Minimally invasive beating-heart ASD closure will avoid using CPB and improve the patient rehabilitation. Reliable visualization of structures within the heart has been one of the major challenges to successful beatingheart surgical interventions [12].

3D Ultrasound (US) is simple, cheap and fast, and allows the surgeon to visualize cardiac structures and instruments through the blood pool. US also has some major disadvantages, being extremely noisy with poor shape definition, which makes it confusing and hard to interpret in the operation room. Tracking tissue in 3D US volumes is particularly difficult due to the low spatial resolution. Therefore, the development of tracking methods for volumetric data in 4D applications is necessary to assist clinical procedures.

An optical flow approach was proposed in [5] to track endocardial surfaces. The data points are initialized manually and a finite element surface is fitted to the points. 
The method extends to 3D the region-based optical flow from [11] using a simple correlation and takes approximately $2 \mathrm{~min} /$ frame after initialization. Boukerroui et al. [2] use a better-adapted similarity measure introduced in [4] to compute region-based optical flow in US image sequences. Their results, although faster, are 2D. The initialization is left out as a separate subject and the authors propose a parameter optimization scheme [2]. A knowledge-based approach using level sets is proposed in [9]. Segmentation and tracking are alternated using shape knowledge, visual information and internal smoothness constraints. The method is parametric and 2D. An interesting 3D cross-correlation-based approach for speckle tracking on simulated data is proposed in [3]. However, 3D speckle tracking poses a serious of difficulties, from optimization and computational costs, to speckle decorrelation in time and space.

A number of approaches have been tried. Block matching is fast and simple, but estimates velocities at low level and lacks robustness. Optical flow has higher sensitivity and specificity, but is very slow and must find a good compromise between local and global displacements.

In this paper, we present an approach adapted to ASD tracking that estimates velocities for an entire block using the concept of voxel-based optical flow. The method is fast and avoids the problems of the traditional block matching, while using the sensitivity of optical flow. The computation is done volumetrically and the displacement optimizes a similarity measure relative to both the previous and original frames. We compare the new results to those obtained by classical implementations of block matching and optical flow.

Section 2 of the paper presents the methodology of the tracking algorithms. We mention block matching, optical flow and similarity measures, and introduce the block flow technique. Comparative results on 3D cardiac US images are shown in Section 3 .

\section{Method}

At this stage, the initialization of the process is done manually by allowing the user to select a block in the first frame that is centered on the ASD. This gives a first estimate of the ASD location and a template for the computation of similarity scores, as seen later in the paper.

\subsection{Block Matching}

The first approach we considered was that of single block matching [8]. This multiscale intensity-based method assumes that there is a global intensity relationship between two images. We are simply interested in matching two blocks, which simplifies the algorithm. We investigated three types of similarity measure suitable for images of the same modality. First, an identity measure, the sum of squared differences $(S S D)$ of normalized intensities, as seen in Equation (1); a linear measure, the inverse Pearson correlation coefficient (PCC), as in Equation (2); and a statistical 
measure, the maximum likelihood (MLE) introduced in [4] and refined in [2], as seen in Equation (3). ref is the reference block, tar the target and $n$ represents the number of voxels in a block. An overview of intensity similarity measures can be found in [10]. While $S S D$ does not cope with relative intensity, $P C C$ minimizes the least square fitting to the original data. The MLE measure [2] assumes that US images are log compressed with Rayleigh noise, but no change of noise between frames. Minimizing the square error between blocks becomes a matter of minimizing the probability distribution function of the additive noise between frames.

$$
\begin{aligned}
& \overline{r e f}=\left(r e f-\mu_{r e f}\right) / \sigma_{r e f} ; \overline{\operatorname{tar}}=\left(\text { tar }-\mu_{t a r}\right) / \sigma_{\text {tar }} ; \\
& S S D=\frac{1}{n} \sum_{i}\left(\overline{\operatorname{ref}}_{i}-\overline{\operatorname{tar}}_{i}\right)^{2} ; \\
& P C C=\frac{1}{n \sum_{i} \overline{r e f}_{i} * \overline{\operatorname{tar}}_{i}} ; \\
& M L E=\frac{1}{n} \sum_{i}\left(\ln \overline{r e f}_{i}-\ln \overline{\operatorname{tar}}_{i}-\ln \left(\exp \left(2\left(\ln \overline{r e f}_{i}-\ln \overline{\operatorname{tar}}_{i}\right)\right)+1\right)\right) ;
\end{aligned}
$$

Our implementation uses two steps, from coarse to fine, and finds the block in the target image that is most similar to the given block in the reference image. To avoid padding at margins, we reduce the size of the reference block to account for clipping at the edge of the target. The best-matched target block is then grown to the original size of the reference.

\subsection{Optical Flow}

As a second approach, we tested the efficiency of region-based optical flow. This is a $3 \mathrm{D}$ extension of the 2D method presented in [2] and based on [11]. There are other techniques to compute optical flow, such as the first-order differential method in [7]. Such methods are very sensitive to noise, which is high in US, and require spatial and temporal smoothing. Our real-time tracking application cannot smooth temporally, as we do not know the data in the next frames. Phase-based techniques [6] require an optimal search space, which is difficult to predict in cardiac images. A review of optical flow techniques can be found in [1].

The major disadvantage of optical flow techniques is the computational load. We propose a two-step multi-scale approach, which uses both block matching and optical flow. Block matching gives the coarse velocity, which is subsequently refined by optical flow. At both levels, we employed $M L E$ as similarity measure. First, the contour of the ASD is extracted in the first frame and then the velocity of the entire block is estimated. At the finer level, the optical flow is estimated only for the contour voxels, which are then wrapped by the resulting block. This approach is considerably faster than multi-scale optical flow. 


\subsection{Block Flow}

Finally, we introduce the notion of "block flow", which uses the velocity estimation of optical flow [11], but for the entire block, instead of every voxel. For a given reference block ref, we define empirically a maximum displacement $m d$ in the new image frame. Within the search space, we compute the estimation error as in Equation (4). Minimizing the energy $E$ is equivalent to minimizing the maximum error in similarity between the target block tar and ref, and between tar and absref, where absref is the user-defined block in the first frame of the 4D sequence. $E$ becomes a value of worst match and reduces velocities $u, v$ and $w$ in directions of blocks that are not similar to both the previous frame and the absolute reference. absref acts as a template ensuring that the solution does not become too different than the initial estimate.

Extending to $3 \mathrm{D}$ the principles presented in [2, 11], we obtain the probability distribution $R$, as in Equation (5), where $\tau$ normalizes the probabilities to sum to 1 . The estimate of the three-directional block velocity $V_{b}$ is presented in Equation (6). Singh [11] minimizes its error through a Gauss-Siedel relaxation algorithm. Unlike [11], we do not use neighborhood information, since the displacement is unique for the entire block. As this enforcement is valid for tracking an object like ASD, this is not applicable to any tracking problem.

$$
\begin{aligned}
& E(u, v, w)=\max (\operatorname{MLE}(\overline{\text { ref }}, \overline{\text { tar }}), \operatorname{MLE}(\overline{\text { absref }}, \overline{\text { tar }})) \\
& R(u, v, w)=\frac{1}{\tau}\left(\exp \left(-\frac{E(u, v, w)-\max (E(u, v, w))}{2 m d^{3}}\right)-1\right) ; \\
& V_{b}=\left(\sum_{u, v, w} R(u, v, w) u, \sum_{u, v, w} R(u, v, w) v, \sum_{u, v, w} R(u, v, w) w\right) ;
\end{aligned}
$$

Once the velocity is calculated, we verify if the new match is on the image margins. If that is the case, we grow the block to ensure that it will propagate with the same size as absref.

\section{Results}

To test the tracking algorithms, we used a database of six 4D time sequences of porcine beating hearts with artificially created ASD. All the US data were acquires invivo with a Sonos 7500 Live 3D Echo scanner (Philips Medical Systems, Andover, MA, USA). The time of acquisition was of $2 \mathrm{~s} / \mathrm{case}$ at a frame rate of 25 volumes/s. At this stage, the implementation of the three tracking algorithms was done in Matlab version 7 (MathWorks, Inc.) on a Pentium IV machine with $1 \mathrm{~GB}$ RAM and $2.40 \mathrm{GHz}$ processor. The image size is $80 \times 80 \times 176 \times 50$.

The performance of the block matching algorithm using two levels (coarse to fine) of combined translation-rotation and $M L E$ is shown on the top row of Figure 1. Each block shown in Figure 1 is a $3 \mathrm{D}$ entity visualized from the right atrium looking into 
the left atrium. The rows present the absolute reference in frame 1 and tracking results after 20, 40 and 50 frames. Judging by the small rotations and the rounder shape of ASD, we also tested block matching using only multi-scale translation. The results are almost identical (with an error of maximum 2 voxels) and the speed is increased to 2 frames/s with $S D D$ and $P C C$ similarity criteria. Using $M L E$ as similarity measure gives more sensitivity to the algorithm, but it is more computationally expensive $(0.5$ frame/s).

The performance of the two level optical flow algorithm (block matching at the coarse level and optical flow at the fine level) was visually more robust than that of simple block matching. Using $M L E$ as similarity criterion, the computational speed was of $1 \mathrm{~min} /$ frame. The middle row in Figure 1 shows tracking results using optical flow.

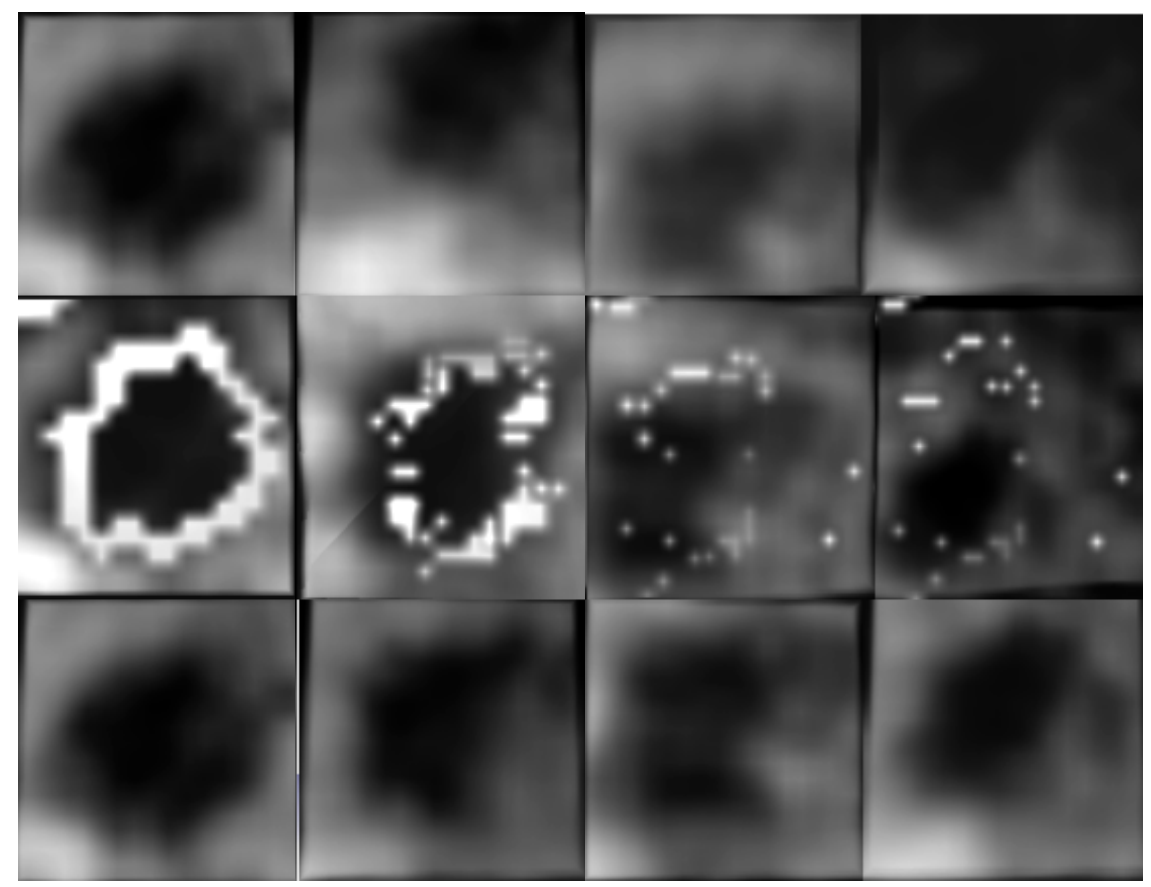

Fig. 1. The comparative performance of the three tracking algorithms. The top row shows results by block matching; the middle row by optical flow; the bottom row by block flow. All three examples used $M L E$ as similarity criterion. From left to right, the columns show 3D blocks visualized from right atrium to left atrium at frames 1, 20, 40 and 50. In the middle row we also show the migration of ASD contour voxels (bright spots), as their velocities are computed by optical flow. While we used smoothing between neighboring points, no connectivity was enforced.

Finally, the bottom row in Figure 1 presents tracking results using our block flow algorithm. Using energy estimation at block level instead of voxel level, the block 
flow method is much faster than optical flow. Its computation speed is 0.3 frame/s, comparable to that of simple block matching, but with much more robust results.

In Figure 2, we present larger 3D volumes (the entire frame) of the results shown in Figure 1. Note that the migration of contour voxels in the optical flow algorithm enlarges the ASD block. Although in Figure 1 the performance of optical flow and block flow look similar and better than that of block matching, Figure 2 shows superior results of our block flow technique.

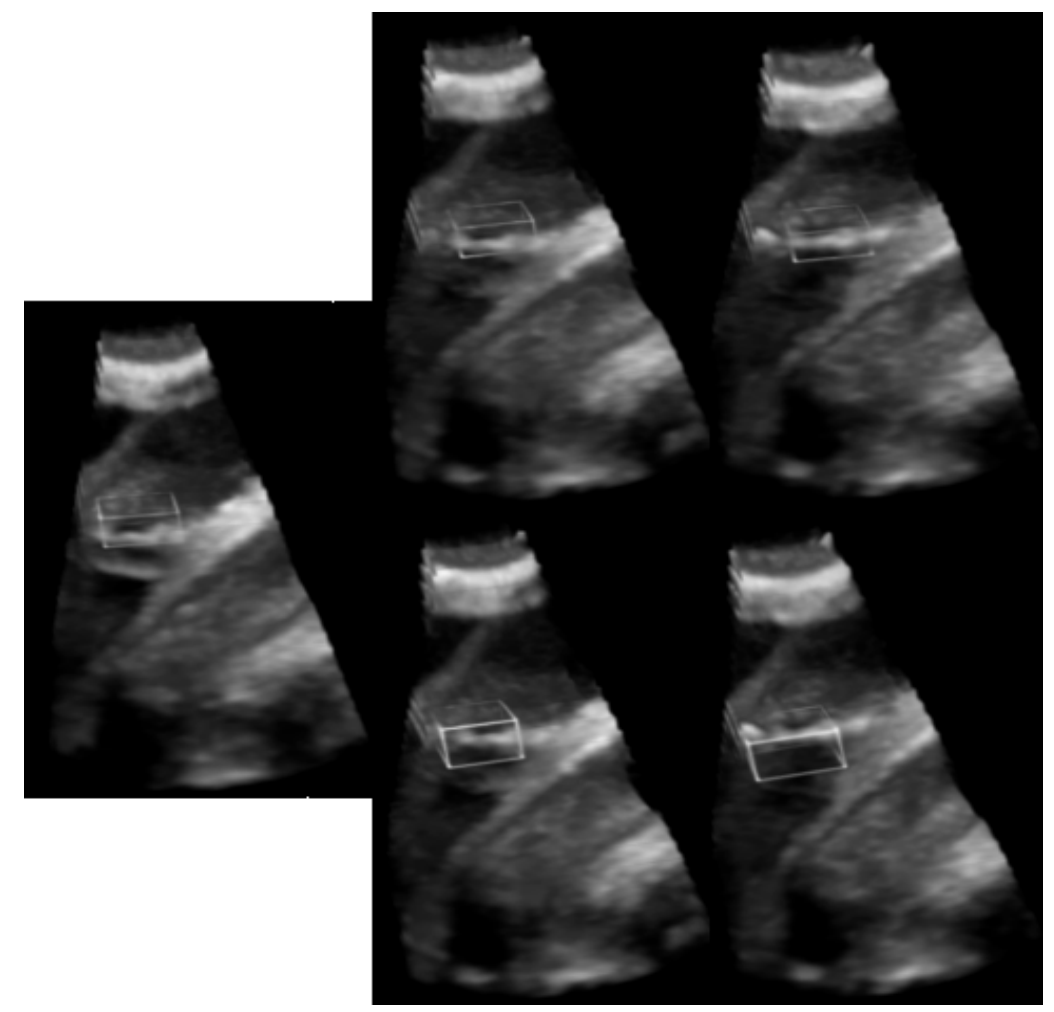

Fig. 2. Comparative tracking results in the full $3 \mathrm{D}$ frame. The left column presents the first frame and the absolute reference marked as a block; the middle column shows tracking results after 25 frames; the right column after 50 frames. The top row shows results by block flow and the bottom row by optical flow. Please note the enlarged blocks on the bottom row.

Table 1 shows tracking quantitative results. Mean $M L E$ scores are computed for the six 4D images using the three tracking algorithms. The minimum score represents the best match. The block flow algorithm performed better than the other two, with the exception of one case, where the optical flow technique had lower scores. Block matching performs better than optical flow over the first 25 frames, due to the error induced by the block growth, but is less stable in time. 
Table 1. The mean $M L E$ scores for the six ASD 4D images using the three tracking techniques. Scores were calculated after 25 and 50 frames as similarities with the absolute reference. $M L E$ scores are normalized and vary from 0 for the perfect match to 100 for the worst. The block flow algorithm performs better in all but one case, where tracking with optical flow has a lower score.

\begin{tabular}{|l|c|c|}
\cline { 2 - 3 } \multicolumn{1}{c|}{} & Frame 25 & Frame 50 \\
\hline Block Matching & 6.82 & 9.29 \\
\hline Optical Flow & 7.46 & 7.17 \\
\hline Block Flow & 4.14 & 5.99 \\
\hline
\end{tabular}

\section{Discussion}

We presented comparative results for the tracking of ASD in sequences of 3D cardiac ultrasound. We introduced a block flow technique, which combined the velocity computation from optical flow with template matching. Enforcing similarity constraints to both the previous and first frames, we ensure optimal and unique solutions. Computing velocity at the block level, our technique is much faster than optical flow and comparable in computation to block matching. Results on in-vivo 4D datasets show that our technique is more stable and has higher sensitivity than both optical flow and block matching in tracking ASD.

The block flow tracking algorithm finds the three-dimensional velocity of an entire block that contains the object of interest. In this application, the object is an ASD with an approximate cylindrical shape. The ASD can be extracted from every new block by simple and fast thresholding.

Compared to the basic block matching algorithm, out method has higher sensitivity in computing displacements, which gives it more robustness in time. Optical flow is sensitive to very localized changes, which makes the method also prone to errors. Interpolation, smoothing and connectivity constraints improve the functioning of optical flow, but also approximate the results. Moreover, the block flow method is much faster than optical flow. For an application such as ASD tracking, the block displacement is sufficient, but this will be too coarse for tracking finer details.

We first normalized the intensities to reduce the effect of noise change and angular reflections from frame to frame. Then we compared the performance of several similarity measures and found that statistical measures, such as maximum likelihood, are better for our application. A coarse to fine approach is desirable for effectiveness and speed.

We used a Rayleigh noise model in the similarity measure. Although we did not smooth the data, the pre-processing of the commercial ultrasound machines may alter the noise distribution. Our assumption led to robust results, but other noise models may be studied.

For future work, we will optimize the algorithm implementation to make it run in real time. The automatic detection of the ASD absolute reference will be investigated along with the use of the cardiac cycle for predictive estimation. 


\section{Acknowledgements}

This work is funded by the National Institutes of Health under grant NIH R01 HL073647-01. The authors would like to thank Dr. Gerald Marx from Children's Hospital in Boston, and Dr. Ivan Salgo from Philips Medical Systems for assistance with image acquisition and informative discussions.

\section{References}

[1] J.L. Barron, D.J. Fleet, S. Beauchemin: Performance of Optical Flow Techniques. In: International Journal of Computer Vision, Vol. 12(1) (1994) 43-77.

[2] D. Boukerroui, J.A. Noble, M. Brady: Velocity Estimation in Ultrasound Images: a Block Matching Approach. In: Information Processing in Medical Imaging (IPMI) (2003) 586598

[3] X. Chen, H. Xie, R. Erkamp, K. Kim, C. Jia, J.M. Rubin, M. O’Donnell: 3-D Correlationbased Speckle Tracking. In: Ultrasonic Imaging. Vol. 27(1) (2005) 21-36

[4] B. Cohen, I. Dinstein: New Maximum Likelihood Motion Estimation Schemes for Noisy Ultrasound Images. In: Pattern Recognition, Vol. 35 (2002) 455-463

[5] Q. Duan, E.D. Angelini, S.L. Herz, C.M. Ingrassia, O. Gerard, K.D. Costa, J.W. Holmes, A.F. Laine: Evaluation of Optical Flow Algorithms for Tracking Endocardial Surfaces on Three-dimensional Ultrasound Data. In: SPIE International Symposium, Medical Imaging Vol. 5750 (2005)

[6] D.J. Fleet, A.D. Jepson: Computation of component image velocity from local phase information. In: International Journal of Computer Vision, Vol. 5(1) (1990) 77-104

[7] B. Lucas, T. Kanade: An Iterative Image Registration Technique with an Application to Stereo Vision. In: Proc. Image Understanding Workshop (1981) 121-130

[8] S. Ourselin, A. Roche, S. Prima, N. Ayache: Block Matching: A General Framework to Improve Robustness of Rigid Registration of Medical Images. In: A.M. DiGioia and S. Delp (eds.): Medical Image Computing and Computer Assisted Intervention (MICCAI 2000), Lectures Notes in Computer Science Vol. 1935 (2000) 557-566

[9] N. Paragios: A Level Set Approach for Shape-driven Segmentation and Tracking of the Left Ventricle. In: IEEE Trans Med Imaging, Vol. 22(6) (2003) 773-786

[10] A. Roche, G. Malandain, N. Ayache: Unifying Maximum Likelihood Approaches in Medical Image Registration. In: International Journal of Imaging Systems and Technology: Special issue on 3D imaging, Vol. 11 (2000) 71-80

[11] A. Singh, P. Allen: Image-flow Computation: an Estimation-theoretic Framework and a Unified Perspective. In: CVGIP: Image Understanding, Vol. 65(2) (1992) 152-177

[12] Y. Suematsu, G.R. Marx, J.A. Stoll, P.E. DuPont, R.O. Cleveland, R.D. Howe, J.K. Triedman, T. Mihaljevic, B.N. Mora, B.J. Savord, I.S. Salgo, P.J. del Nido: ThreeDimensional Echocardiography-guided Beating-heart Surgery without Cardiopulmonary Bypass: a Feasibility Study. In: Journal of Thoracic and Cardiovascular Surgery, Vol. 128(4) (2204) 579-587 